\title{
Optical Absorption of an Interacting Many-Polaron Gas
}

\author{
J. Tempere, J. T. Devreese \\ Departement Natuurkunde, Universiteit Antwerpen (UIA), Universiteitsplein 1, B2610 \\ Antwerpen, Belgium.
}

$(17 / 11 / 2000)$

\begin{abstract}
The optical absorption of a many (continuum) polaron gas is derived in the framework of a variational approach at zero temperature and weak or intermediate electron-phonon coupling strength. We derive a compact formula for the optical conductivity of the many-polaron system taking into account many-body effects in the electron or hole system. Within the method presented here, these effects are contained completely in the dynamical structure factor of the electron or hole system. This allows to build on well-established studies of the interacting electron gas. Based on this approach a novel feature in the absorption spectrum of the many-polaron gas, related to the emission of a plasmon together with a phonon, is identified. As an application and illustration of the technique, we compare the theoretical many-polaron optical absorption spectrum as derived in the present work with the 'd-band' absorption feature in $\mathrm{Nd}_{2} \mathrm{CuO}_{2}$. Similarities are shown between the theoretically and the experimentally derived first frequency moment of the optical absorption of a family of differently doped $\mathrm{Nd}_{2-x} \mathrm{Ce}_{x} \mathrm{CuO}_{4-y}$ materials.
\end{abstract}




\section{INTRODUCTION}

As is the case for polar semiconductors and ionic crystals [1], insight into the nature of polarons in high-temperature superconductors can be gained by studying the optical properties of these materials. The goal of the present paper is to present a theory of the optical conductivity of a system of continuum polarons at any density, including manybody effects between the constituent charge carriers, for small and intermediate values of the electron-phonon coupling constant and for zero temperature. The method we develop here is based on the variational method introduced by Lemmens, Devreese, Brosens (LDB) [2] for the ground state energy of the many-polaron gas. The advantage of this approach over other theories of many-polaron optical absorption [3, 4] is that it allows to include the many-body effects in the system of the constituent charge carriers of the polaron gas on the level of the dynamical structure factor of the underlying electron (or hole) system. Thus it is possible to select the level of approximation used in the treatment of the many-polaron gas by choosing the appropriate expression of the dynamical structure factor for the electron (or hole) system.

Recently the infrared spectrum of cuprates has been the subject of intensive investigations [5 13], especially in the case of the neodymium-cerium cuprate family

$\mathrm{Nd}_{2-x} \mathrm{Ce}_{x} \mathrm{CuO}_{4-y}$ (NCCO) [5,8-13]. Several optical absorption features in the infrared cuprate spectrum have been tentatively associated with large polarons [0],14] or with a mixture of large and small (bi)polarons [15]. These comparisons with polaron theory were derived using a single-polaron picture, so that the density (doping) dependence of the optical absorption spectra could not be studied in detail. The many-body theory of the $N$-polaron spectrum, presented here, allows to study the density (doping) dependence of optical absorption spectra. As a first application of the many-polaron optical absorption theory introduced here, a preliminary comparison is presented between the theoretical many-polaron optical absorption derived in the current work and the mid-infrared spectrum of the neodymiumcerium cuprates recently determined experimentally by Calvani and co-workers [5]. 


\section{OPTICAL ABSORPTION IN THE MANY-POLARON SYSTEM}

\section{A. The LDB variational wave function for a many-polaron system}

The Hamiltonian of a system of $N$ interacting continuum polarons is given by:

$H_{0}=\sum_{j=1}^{N} \frac{p_{j}^{2}}{2 m_{b}}+\sum_{\mathbf{k}} \hbar \omega_{\mathrm{LO}} a_{\mathbf{k}}^{+} a_{\mathbf{k}}+\sum_{\mathbf{k}} \sum_{j=1}^{N}\left[e^{i \mathbf{k} \cdot \mathbf{r}_{j}} a_{\mathbf{k}} V_{\mathbf{k}}+e^{-i \mathbf{k} \cdot \mathbf{r}_{j}} a_{\mathbf{k}}^{+} V_{\mathbf{k}}^{*}\right]+\frac{e^{2}}{2 \varepsilon_{\infty}} \sum_{j=1}^{N} \sum_{\ell(\neq j)=1}^{N} \frac{1}{\left|\mathbf{r}_{i}-\mathbf{r}_{j}\right|}$

where $\mathbf{r}_{j}, \mathbf{p}_{j}$ represent the position and momentum of the $N$ constituent electrons (or holes) with band mass $m_{b} ; a_{\mathbf{k}}^{+}, a_{\mathbf{k}}$ denote the creation and annihilation operators for longitudinal optical (LO) phonons with wave vector $\mathbf{k}$ and frequency $\omega_{\mathrm{LO}} ; V_{\mathbf{k}}$ describes the amplitude of the interaction between the electrons and the phonons; and $e$ is the elementary electron

charge. The ground state energy of this many-polaron Hamiltonian has been studied before by LDB [2], for weak and intermediate strengths of the electron-phonon coupling, by introducing a variational wave function:

$$
\left|\psi_{\mathrm{LDB}}\right\rangle=U|\phi\rangle\left|\varphi_{\mathrm{el}}\right\rangle
$$

where $\left|\varphi_{\mathrm{el}}\right\rangle$ represents the ground-state many-body wave function for the electron (or hole) system and $|\phi\rangle$ is the phonon vacuum, and $U$ is a many-body unitary operator which determines a canonical transformation for a fermion gas interacting with a boson field:

$$
U=\exp \left\{\sum_{j=1}^{N} \sum_{\mathbf{k}}\left(f_{\mathbf{k}} a_{\mathbf{k}} e^{i \mathbf{k} \cdot \mathbf{r}_{j}}-f_{\mathbf{k}}^{*} a_{\mathbf{k}}^{+} e^{-i \mathbf{k} \cdot \mathbf{r}_{j}}\right)\right\} .
$$

In the limit of one fermion, $U$ reduces to a canonical transformation inspired by Tomonaga [16] and applied later by several workers, after Lee, Low and Pines [17], but always for one particle-theories. In LDB [2], this canonical transformation was extended and used to establish a many-fermion theory. The $f_{\mathbf{k}}$ were determined variationally [2] resulting in

$$
f_{\mathbf{k}}=\frac{V_{\mathbf{k}}}{\hbar \omega_{L O}+\frac{\hbar^{2} k^{2}}{2 m_{b} S(\mathbf{k})}}
$$


for a system with total momentum $\mathbf{P}=\sum_{j} \mathbf{p}_{j}=0$. In this expression, $S(\mathbf{k})$ represents the static structure factor of the constituent interacting many electron or hole system :

$$
N S(\mathbf{k})=\left\langle\sum_{j=1}^{N} \sum_{j^{\prime}=1}^{N} e^{i \mathbf{k} \cdot\left(\mathbf{r}_{j}-\mathbf{r}_{j^{\prime}}\right)}\right\rangle .
$$

The angular brackets $\langle\ldots\rangle$ represent the expectation value with respect to the ground state. It may be emphasized that (3), although it appears like a straightforward generalization of the one-particle transformation in [16], represents -especially in its implementation- a nontrivial extension of a one-particle approximation to a many-body system. As noted in the introduction, the main advantage of the LDB many-polaron variational approach lies in the fact that the many-body effects in the system of charge carriers (electrons or holes) are completely contained in the structure factor of the electron (or hole) gas. This advantage will be carried through into the calculation of the optical properties of the interacting gas of continuum polarons which is the subject of the current paper.

\section{B. Kubo formula for the optical conductivity of the many-polaron gas}

The many-polaron optical conductivity is the response of the current-density, in the system described by the Hamiltonian (11), to an applied electric field (along the $x$-axis) with frequency $\omega$. This applied electric field introduces a perturbation term in the Hamiltonian (更), which couples the vector potential of the incident electromagnetic field to the currentdensity. As is well known, within linear response theory, the optical conductivity can be expressed through the Kubo formula as a current-current correlation function [18]:

$$
\sigma(\omega)=i \frac{N e^{2}}{\mathrm{~V} m_{b} \omega}+\frac{1}{\mathrm{~V} \hbar \omega} \int_{0}^{\infty} e^{i \omega t}\left\langle\left[J_{x}(t), J_{x}(0)\right]\right\rangle d t
$$

In this expression, $\mathrm{V}$ is the volume of the system, and $J_{x}$ is the $x$-component of the current operator $\mathbf{J}$, which is related to the momentum operators of the charge carriers:

$$
\mathbf{J}=\frac{q}{m_{b}} \sum_{j=1}^{N} \mathbf{p}_{j}=\frac{q}{m_{b}} \mathbf{P}
$$


with $q$ the charge of the charge carriers ( $+e$ for holes, $-e$ for electrons) and $\mathbf{P}$ the total momentum operator of the charge carriers. The real part of the optical conductivity at temperature zero, which is proportional to the optical absorption coefficient, can be written as a function of the total momentum operator of the charge carriers as follows :

$$
\operatorname{Re}[\sigma(\omega)]=\frac{1}{\mathrm{~V} \hbar \omega} \frac{e^{2}}{m_{b}^{2}} \operatorname{Re}\left\{\int_{0}^{\infty} e^{i \omega t}\left\langle\left[P_{x}(t), P_{x}(0)\right]\right\rangle d t\right\} .
$$

Integrating by parts twice, the real part of the optical conductivity of the many-polaron system can be written with a force-force correlation function:

$$
\operatorname{Re}[\sigma(\omega)]=\frac{1}{\mathrm{~V} \hbar \omega^{3}} \frac{e^{2}}{m_{b}^{2}} \operatorname{Re}\left\{\int_{0}^{\infty} e^{i \omega t}\left\langle\left[F_{x}(t), F_{x}(0)\right]\right\rangle d t\right\},
$$

with $\mathbf{F}=(i / \hbar)\left[H_{0}, \mathbf{P}\right]$. The commutator of the Hamiltonian (1) with the total momentum operator of the charge carriers simplifies to

$$
\mathbf{F}=-i \sum_{\mathbf{k}} \sum_{j=1}^{N} \mathbf{k}\left(e^{i \mathbf{k} \cdot \mathbf{r}_{j}} a_{\mathbf{k}} V_{\mathbf{k}}+e^{-i \mathbf{k} \cdot \mathbf{r}_{j}} a_{\mathbf{k}}^{+} V_{\mathbf{k}}^{*}\right) .
$$

This result for the force operator clarifies the significance of using the force-force correlation function rather than the momentum-momentum correlation function. The operator product $F_{x}(t) F_{x}(0)$ is proportional to $\left|V_{\mathbf{k}}\right|^{2}$, the charge carrier - phonon interaction strength. This will be a distinct advantage for any expansion of the final result in the charge carrier -

phonon interaction strength, since one power of $\left|V_{\mathbf{k}}\right|^{2}$ is factored out beforehand. Denoting $\rho_{\mathbf{k}}=\sum_{j=1}^{N} e^{i \mathbf{k} \cdot \mathbf{r}_{j}}$, the real part of the optical conductivity takes the form:

$$
\operatorname{Re}[\sigma(\omega)]=\frac{1}{\mathrm{~V} \hbar \omega^{3}} \frac{e^{2}}{m_{b}^{2}} \operatorname{Re}\left\{\sum_{\mathbf{k}, \mathbf{k}^{\prime}} k_{x} \cdot k_{x}^{\prime} \int_{0}^{\infty} e^{i \omega t}\left\langle\left[\begin{array}{c}
e^{i H_{0} t / \hbar}\left(\rho_{\mathbf{k}} a_{\mathbf{k}} V_{\mathbf{k}}+\rho_{-\mathbf{k}} a_{\mathbf{k}}^{+} V_{\mathbf{k}}^{*}\right) e^{-i H_{0} t / \hbar}, \\
\left(\rho_{\mathbf{k}^{\prime}} a_{\mathbf{k}^{\prime}} V_{\mathbf{k}^{\prime}}+\rho_{-\mathbf{k}^{\prime}} a_{\mathbf{k}^{\prime}}^{+} V_{\mathbf{k}^{\prime}}^{*}\right)
\end{array}\right]\right)_{0} d t\right\}
$$

Up to this point, no approximations other than linear response theory have been made.

\section{LDB canonical transformation for the optical conductivity}

The expectation value appearing in the right hand side of expression (11] for the real part of the optical conductivity is calculated now with respect to the LDB many-polaron 
wave function $\left|\psi_{\mathrm{LDB}}\right\rangle(2)$ :

$$
\begin{aligned}
\mathcal{J}\left(\mathbf{k}, \mathbf{k}^{\prime}\right) & =\left\langle\psi_{\mathrm{LDB}}\left|\left[e^{i H_{0} t / \hbar}\left(\rho_{\mathbf{k}} a_{\mathbf{k}} V_{\mathbf{k}}+\rho_{-\mathbf{k}} a_{\mathbf{k}}^{+} V_{\mathbf{k}}^{*}\right) e^{-i H_{0} t / \hbar},\left(\rho_{\mathbf{k}^{\prime}} a_{\mathbf{k}^{\prime}} V_{\mathbf{k}^{\prime}}+\rho_{-\mathbf{k}^{\prime}} a_{\mathbf{k}^{\prime}}^{+} V_{\mathbf{k}^{\prime}}^{*}\right)\right]\right| \psi_{\mathrm{LDB}}\right\rangle \\
& =\left\langle\varphi_{\mathrm{el}}\right|\left\langle\phi\left|\left[\begin{array}{c}
\left.e^{i H^{\prime} t / \hbar} U^{-1}\left(\rho_{\mathbf{k}} a_{\mathbf{k}} V_{\mathbf{k}}+\rho_{-\mathbf{k}} a_{\mathbf{k}}^{+} V_{\mathbf{k}}^{*}\right) U e^{-i H^{\prime} t / \hbar},\right]|\phi\rangle\left|\varphi_{\mathrm{el}}\right\rangle \\
U^{-1}\left(\rho_{\mathbf{k}^{\prime}} a_{\mathbf{k}^{\prime}} V_{\mathbf{k}^{\prime}}+\rho_{-\mathbf{k}^{\prime}} a_{\mathbf{k}^{\prime}}^{+} V_{\mathbf{k}^{\prime}}^{*}\right) U
\end{array}\right]\right|\right.
\end{aligned}
$$

where $U$ is the many-polaron canonical transformation defined in (3) and $H^{\prime}=U^{-1} H_{0} U$ is the transformed Hamiltonian obtained in [2]. The canonical transformation of the force term is

$$
U^{-1}\left(\rho_{\mathbf{k}} a_{\mathbf{k}} V_{\mathbf{k}}+\rho_{-\mathbf{k}} a_{\mathbf{k}}^{+} V_{\mathbf{k}}^{*}\right) U=\rho_{\mathbf{k}}\left(a_{\mathbf{k}}-f_{\mathbf{k}}^{*} \rho_{-\mathbf{k}}\right) V_{\mathbf{k}}+\rho_{-\mathbf{k}}\left(a_{\mathbf{k}}^{+}-f_{\mathbf{k}} \rho_{\mathbf{k}}\right) V_{\mathbf{k}}^{*}
$$

The terms of lowest order in the electron-phonon interaction amplitude $\left|V_{\mathbf{k}}\right|^{2}$ are given by

$$
\begin{aligned}
\mathcal{J}\left(\mathbf{k}, \mathbf{k}^{\prime}\right) & =\left|V_{\mathbf{k}}\right|^{2} \delta_{\mathbf{k k}^{\prime}}\left\langle\varphi_{\mathrm{el}}\left|\left\langle\phi\left|e^{i H^{\prime} t / \hbar} \rho_{\mathbf{k}} a_{\mathbf{k}} e^{-i H^{\prime} t / \hbar} \rho_{-\mathbf{k}} a_{\mathbf{k}}^{+}-\rho_{\mathbf{k}} a_{\mathbf{k}} e^{i H^{\prime} t / \hbar} \rho_{-\mathbf{k}} a_{\mathbf{k}}^{+} e^{-i H^{\prime} t / \hbar}\right| \phi\right\rangle\right| \varphi_{\mathrm{el}}\right\rangle \\
& =2 i\left|V_{\mathbf{k}}\right|^{2} \delta_{\mathbf{k k}^{\prime}} \operatorname{Im}\left[\left\langle\varphi_{\mathrm{el}}\left|\left\langle\phi\left|e^{i H^{\prime} t / \hbar} \rho_{\mathbf{k}} a_{\mathbf{k}} e^{-i H^{\prime} t / \hbar} \rho_{-\mathbf{k}} a_{\mathbf{k}}^{+}\right| \phi\right\rangle\right| \varphi_{\mathrm{el}}\right\rangle\right] .
\end{aligned}
$$

Taking the expectation value with respect to the phonon vacuum, we find

$$
\mathcal{J}\left(\mathbf{k}, \mathbf{k}^{\prime}\right)=2 i\left|V_{\mathbf{k}}\right|^{2} \delta_{\mathbf{k} \mathbf{k}^{\prime}} \operatorname{Im}\left\{e^{-i \omega_{\mathrm{LO}} t}\left\langle\varphi_{\mathrm{el}}\left|e^{i H^{\prime} t / \hbar} \rho_{\mathbf{k}} e^{-i H^{\prime} t / \hbar} \rho_{-\mathbf{k}}\right| \varphi_{\mathrm{el}}\right\rangle\right\}
$$

This can be substituted in the expression (11) for the real part of the optical conductivity, which becomes

$$
\operatorname{Re}[\sigma(\omega)]=-2 \frac{1}{\mathrm{~V} \hbar \omega^{3}} \frac{e^{2}}{m_{b}^{2}} \operatorname{Im}\left\{\sum_{\mathbf{k}} k_{x}^{2}\left|V_{\mathbf{k}}\right|^{2} \int_{0}^{\infty} e^{i \omega t} \operatorname{Im}\left[e^{-i \omega_{\mathrm{LO}} t}\left\langle\varphi_{\mathrm{el}}\left|e^{i H^{\prime} t / \hbar} \rho_{\mathbf{k}} e^{-i H^{\prime} t / \hbar} \rho_{-\mathbf{k}}\right| \varphi_{\mathrm{el}}\right\rangle\right] d t\right\} .
$$

The right hand side of $(18)$ can be written in a more compact form by introducing the dynamical structure factor of the electron (or hole) system.

\section{General expression}

To find the formula for the real part of the optical conductivity in its final form, we introduce the standard expression for the dynamical structure factor of the system of charge carriers interacting through a Coulomb potential, 


$$
S(\mathbf{q}, w)=\int_{-\infty}^{+\infty}\left\langle\varphi_{\mathrm{el}}\left|\frac{1}{2} \sum_{j, \ell} e^{i \mathbf{q} \cdot\left(\mathbf{r}_{j}(t)-\mathbf{r}_{\ell}(0)\right)}\right| \varphi_{\mathrm{el}}\right\rangle e^{i w t} d t
$$

Rewriting expression (18) with the dynamical structure factor of the electron (or hole) gas results in:

$$
\operatorname{Re}[\sigma(\omega)]=\frac{n}{\hbar \omega^{3}} \frac{e^{2}}{m_{b}^{2}} \sum_{\mathbf{k}} k_{x}^{2}\left|V_{\mathbf{k}}\right|^{2} S\left(\mathbf{k}, \omega-\omega_{\mathrm{LO}}\right),
$$

where $n=N / \mathrm{V}$ is the density of charge carriers. As noted before, $V_{\mathbf{k}}$ is the electron-phonon interaction amplitude and $k_{x}$ is the $x$-component of the wave vector. Formula (20) for the optical absorption of the many-polaron system has an intuitively appealing form.

In the theory of one-polaron optical absorption for weak coupling constants $\alpha$, the optical absorption coefficient as obtained from Fermi's golden rule is [19]:

$$
1 \text { polaron : } \operatorname{Re}[\sigma(\omega)] \propto \omega^{-3} \sum_{\mathbf{k}} k_{x}^{2}\left|V_{\mathbf{k}}\right|^{2} \delta\left[\hbar k^{2} /\left(2 m_{\mathrm{b}}\right)-\left(\omega-\omega_{\mathrm{LO}}\right)\right]
$$

At low densities, the dynamical structure factor $S(q, \nu)$ is strongly peaked around $q^{2} / 2=\nu$ and is close to zero everywhere else [20]. Substituting a delta-function $\delta\left(q^{2} / 2-\nu\right)$ for the dynamical structure factor in formula (20) it is easily seen that the one-polaron limit (21) [19] is retrieved. The one-polaron result (21) is derived [19] by considering a process in which the initial state consists of a photon of energy $\hbar \omega$ and a polaron in its ground state, and the final state consists of an emitted LO phonon with energy $\hbar \omega_{\text {LO }}$ and the polaron, scattered into a state with momentum $\mathbf{k}$ and kinetic energy $(\hbar k)^{2} /\left(2 m_{\mathrm{b}}\right)=\hbar\left(\omega-\omega_{\mathrm{LO}}\right)$. The many-polaron result, formula (20), is a generalization of this one-polaron picture. The contribution which corresponds to the scattering of a polaron into the momentum state $\mathbf{k}$ and energy $\hbar\left(\omega-\omega_{\mathrm{LO}}\right)$ is now weighed by the dynamical structure factor $S\left(k, \omega-\omega_{\mathrm{LO}}\right)$ of the electron (or hole) gas.

Formula (20) is reminiscent of the Hopfield formula [21] describing the effect of impurities on the optical absorption of metals, which in turn is related to the expression obtained by Ron and Tzoar [22] for the optical absorption in a quantum plasma. Formula (20) also represents a generalization of the results obtained by Gurevich, Lang and Firsov 23. These 
authors focused their attention on the many-body effects related to the Fermi exclusion statistics, whereas the present analysis will extend the results of [23] to study the influence of plasmons and further many-body effects in the system of the constituent electrons or holes, as discussed in the next section.

The advantage of the LDB canonical transformation method [2] for the evaluation of the ground state energy of a polaron gas is that the many-body effects are contained in the static structure factor of the electron (or hole) system, appearing in the analytical expression for the energy. The corresponding advantage of the canonical transformation in the present case, for the optical conductivity, is that many-body effects are again incorporated through a structure factor, now the dynamical structure factor of the electron (or hole) system. The level of approximation made in treating the many-body nature of the polaron system is determined by the choice of the dynamical structure factor of the electron or hole system.

In the present treatment of the electron-phonon interactions, the terms to leading order in $\left|V_{\mathbf{k}}\right|^{2}$ are automatically taken into account through the variational formulation based on LDB [2]. As noted before, the use of the force-force correlation function allows to express the real part of the optical conductivity as $\operatorname{Re}[\sigma(\omega)]=\alpha \mathcal{F}(\omega, \alpha)$, so that to lowest order

in $\alpha, \operatorname{Re}[\sigma(\omega)]=\alpha \mathcal{F}(\omega, \alpha=0)$ where the electron-phonon interaction is no longer present in the factor $\mathcal{F}$ which includes the many-body effects of the electron (or hole) system. A possible way to take into account higher-order terms in the electron-phonon interactions would be to include electron-phonon coupling effects at the level of the dynamical structure factor appearing in $\mathcal{F}$, in a manner similar to Mahan's treatment of the polaron spectral function [24], or to include multiple-phonon final states in the calculation [25].

\section{E. Scaling relation for the optical absorption in two and three dimensions}

The modulus squared of the Fröhlich electron-phonon interaction amplitude is given by 


$$
\left|V_{\mathbf{k}}\right|^{2}=\left\{\begin{array}{l}
\frac{\left(\hbar \omega_{\mathrm{LO}}\right)^{2}}{k^{2}} \frac{4 \pi \alpha}{\mathrm{V}} \sqrt{\frac{\hbar}{2 m_{b} \omega_{\mathrm{LO}}}} \text { in } 3 \mathrm{D} \\
\frac{\left(\hbar \omega_{\mathrm{LO}}\right)^{2}}{k} \frac{2 \pi \alpha}{\mathrm{A}} \sqrt{\frac{\hbar}{2 m_{b} \omega_{\mathrm{LO}}}} \text { in } 2 \mathrm{D}
\end{array}\right.
$$

where $\alpha$ is the (dimensionless) Fröhlich coupling constant determining the coupling strength between the charge carriers and the longitudinal optical phonons, and $\mathrm{A}$ is the surface of the $2 \mathrm{D}$ system [26]. In what follows, we will use polaron units $\left(\hbar=m_{b}=\omega_{\mathrm{LO}}=1\right)$. The sum over wave vectors in (20) can be written as an integral, so that for the three dimensional case (with dynamical structure factor $S_{3 \mathrm{D}}$ ) we find:

$$
\operatorname{Re}\left[\sigma_{3 \mathrm{D}}(\omega)\right]=n e^{2} \frac{2}{3} \alpha \frac{1}{2 \pi \omega^{3}} \int_{0}^{\infty} d q q^{2} S_{3 \mathrm{D}}\left(q, \omega-\omega_{\mathrm{LO}}\right),
$$

and for the two-dimensional case:

$$
\operatorname{Re}\left[\sigma_{2 \mathrm{D}}(\omega)\right]=n e^{2} \frac{\pi}{2} \alpha \frac{1}{2 \pi \omega^{3}} \int_{0}^{\infty} d q q^{2} S_{2 \mathrm{D}}\left(q, \omega-\omega_{\mathrm{LO}}\right) .
$$

From these expressions, it is clear that the scaling relation

$$
\operatorname{Re}\left[\sigma_{2 \mathrm{D}}(\omega, \alpha)\right]=\operatorname{Re}\left[\sigma_{3 \mathrm{D}}(\omega, 3 \pi \alpha / 4)\right]
$$

which holds for the one-polaron case introduced in ref. [26], is also valid for the many-polaron case if the corresponding 2D or 3D dynamical structure factor is used.

\section{RESULTS AND DISCUSSION}

\section{A. General results}

The expressions $(23,24)$ allow us to derive results both for a three-dimensional and for a

two dimensional polaron gas at $T=0$. The choice of a dynamical structure factor for the electron (or hole) system allows furthermore to study the different levels of approximation (Hartree-Fock, RPA,...) in the treatment of the many-electron or many-hole system. The results presented in this section were obtained using the material parameters of GaAs (for the 
two-dimensional case) and $\mathrm{ZnO}$ (for the three-dimensional case). These material parameters 8,27 29 are summarized in Table I.

Fig. 1 shows the Hartree-Fock and the RPA result for the 2D many-polaron optical absorption spectrum (for GaAs, at a density $n=10^{12} \mathrm{~cm}^{-2}$ ). For reference, the dashed curve represents the familiar one-polaron result. In a first step, we discuss the result obtained by using the Hartree-Fock expression for the dynamical structure factor of the electron (or hole) system in the expressions (23,24).

The Fermi statistics cause the polarons to fill up a Fermi sphere up to $k_{\mathrm{F}}=[n /(2 \pi)]^{1 / 2}$. The optical absorption of the polaron gas resulting from this system is represented by the full curve labeled 'Hartree-Fock' in Fig. 1. The spectral weight at frequencies between $\omega_{\text {LO }}$ and $1.4 \omega_{\mathrm{LO}}$ in Fig. 1 is reduced as compared to the single polaron case, whereas at higher frequencies it is enhanced. A kink appears in the spectrum at $\omega=\omega_{\mathrm{LO}}+E_{\mathrm{F}} / \hbar$, as indicated by the dotted vertical line in Fig. 1.

This can be understood as follows. The absorption process is characterized by an initial state consisting of a polaron gas filling up the Fermi sphere (up to energy $E_{\mathrm{F}}$, at $T=0$ ) and a photon with given energy $\hbar \omega$, and by a final state made up of an emitted LO phonon with energy $\hbar \omega_{\mathrm{LO}}$ and a polaron gas such that one polaron state inside the Fermi sphere is not occupied and one polaron state with energy $E>E_{\mathrm{F}}$ is occupied. The incident photon can only excite polarons out of the Fermi sea for which $\hbar \omega>h \omega_{\mathrm{LO}}+E_{\mathrm{F}}$. A straightforward calculation in $2 \mathrm{D}$ shows that the fraction of polaron states in the Fermi sphere which can interact with a photon of energy $\hbar \omega$ is given by

$$
\left\{\begin{array}{l}
0 \text { for } \omega<\omega_{\mathrm{LO}} \\
\frac{\hbar\left(\omega-\omega_{\mathrm{LO}}\right)}{E_{\mathrm{F}}} \text { for } \omega_{\mathrm{LO}}<\omega<E_{\mathrm{F}} / \hbar+\omega_{\mathrm{LO}} \\
1 \text { for } \omega>E_{\mathrm{F}} / \hbar+\omega_{\mathrm{LO}}
\end{array}\right.
$$

For photon frequencies between $\omega_{\mathrm{LO}}$ and $\omega_{\mathrm{LO}}+E_{\mathrm{F}} / \hbar$, the number of polarons which cannot participate in the optical absorption process due to the Pauli exclusion principle, decreases linearly. At $\omega=\omega_{\mathrm{LO}}+E_{\mathrm{F}} / \hbar$, all polarons can participate. This leads to a kink in the 
function (26) describing the number of polarons which can interact with the photon of given energy $\hbar \omega$, as a function of $\omega$. This is also the origin of the kink in the optical absorption. This kink in the $2 \mathrm{D}$ many-polaron optical absorption spectrum at $\hbar \omega=\hbar \omega_{\mathrm{LO}}+E_{\mathrm{F}}$ was already noted in [27].

The full curve labeled with 'RPA' in Fig. 1 is obtained by using the random phase approximation (RPA) for the dynamical structure factor of the electron (or hole) system. It illustrates the combined effects of the Fermi statistics, discussed in the previous paragraph, and screening in the electron (or hole) system. In comparison to the Hartree-Fock curve, the main effect is an overall reduction of the spectral weight at frequencies $\omega>\omega_{\mathrm{LO}}$. There is however a second effect, which is the appearance of a contribution related to plasmons this is the subject of the next subsection.

\section{B. Plasmon-phonon contribution}

The RPA dynamical structure factor for the electron (or hole) system can be separated in two parts, one related to continuum excitations of the electrons (or holes) $S_{\text {cont }}$, and one related to the undamped plasmon branch [30]:

$$
S_{\mathrm{RPA}}(q, \omega)=A_{\mathrm{pl}}(q) \delta\left[\omega-\omega_{\mathrm{pl}}(q)\right]+S_{\mathrm{cont}}(q, \omega),
$$

where $\omega_{\mathrm{pl}}(q)$ is the wave number dependent plasmon frequency and $A_{\mathrm{pl}}$ is the strength of the undamped plasmon branch [30]. The insets of Fig. 2 depict the regions in the $q-v$ plane $\left(q=k / k_{F}, v=m_{\mathrm{b}} \omega /\left(\hbar k_{F}^{2}\right)\right)$ where the RPA dynamical structure factor is different from zero. The contribution (after substitution of $S_{\mathrm{RPA}}$ in (23)-(24)) in the many-polaron optical absorption) deriving from the undamped plasmon branch $A_{\mathrm{pl}}(q) \delta\left[\omega-\omega_{\mathrm{pl}}(q)\right]$ will be denoted as 'plasmon-phonon' contribution. The physical process related to this contribution is the emission of both a phonon and a plasmon in the scattering process.

Figure 2 shows the result for the optical absorption of the many-polaron gas for the 2D case (GaAs, left panel) and the 3D case ( $\mathrm{ZnO}$, right panel). For reference, the dashed curves 
show the one-polaron result. The full curves show the many-polaron results in the randomphase approximation. The shaded gray areas indicate the plasmon-phonon contribution.

Now examine the 3D case (the right panel of Fig. 2). The frequency of the undamped plasmon mode lies between $\omega_{1}$ and $\omega_{2}$ where $\omega_{1}=\omega_{\mathrm{pl}}=\sqrt{4 \pi n e^{2} / m_{\mathrm{b}}}$ is the frequency of the plasmon branch at $q=0$, and $\omega_{2}$ is the frequency at which the branch of the undamped plasmons enters the Landau damping region (whose edge is given by $\omega=\hbar q^{2} /\left(2 m_{b}\right)+$ $\left.\hbar k_{\mathrm{F}} q /\left(2 m_{b}\right)\right)$. The corresponding plasmon-phonon contribution to the optical absorption 'starts' at $\omega_{\mathrm{LO}}+\omega_{1}$ and 'ends' at $\omega_{\mathrm{LO}}+\omega_{2}$. These frequencies are indicated by vertical dotted lines in the right panel of Fig. 2.

In the $2 \mathrm{D}$ case, the undamped plasmon branch is acoustic-like; for $q \rightarrow 0, \omega_{\mathrm{pl}} \rightarrow 0$. Consequently, the phonon-plasmon peak in this case extends from $\omega_{\mathrm{LO}}$ up to $\omega_{\mathrm{LO}}+\omega_{2}$ where $\omega_{2}$ is the frequency at which the undamped plasmon branch enters the region of the continuum excitations of the 2D (RPA) electron gas.

In Fig. 3, the evolution of the many-polaron optical absorption spectrum is shown as the density of electrons (or holes) is increased. Two effects can be observed for increasing density: the reduction of the optical absorption above $\omega>\omega_{\mathrm{LO}}$ and the shift towards higher frequencies of the plasmon-phonon contribution, both in 2D (left panel) and in 3D (right panel). The f-sum rule is nevertheless satisfied due to the presence of a central $\delta(\omega)$ peak 31] in the optical absorption of the polaron gas at $T=0$.

\section{Comparison with other theories}

In earlier work, Wu, Peeters and Devreese [27] studied the influence of screening on the electron-phonon interaction in a two-dimensional electron gas based on a memory function approach using a perturbation expansion in the electron-LO phonon coupling constant. The results of this perturbative approach of 27] for the optical conductivity in 2D, also in the RPA framework, are consistent with the results derived from the present method based on

the variational LDB unitary transformation. These authors found an enhancement of the 
optical absorption at the frequency where the undamped plasmon branch reaches the region of continuum excitations of the electron gas. The present, variational, method extends these results by taking into account the entire undamped plasmon branch.

Recently, Cataudella, De Filippis and Iadonisi [3] investigated the optical properties of the many-polaron gas by calculating the correction due to electron-phonon interactions to the RPA dielectric function of the electron gas, starting from the Feynman polaron model and ref. [1]. An aspect of the present method is that it is not restricted to the random-phase approximation for the treatment of the many-body effects between the charge carriers. Cataudella et al. [3] also find a suppression of the optical absorption with increasing density. To our knowledge, the plasmon-phonon contribution was not revealed by the work of Cataudella et al. [3].

\section{Comparison to the infrared spectrum of $\mathrm{Nd}_{2-x} \mathrm{Ce}_{x} \mathrm{CuO}_{2-y}$}

Calvani and collaborators have performed doping-dependent measurements of the infrared absorption spectra of the high- $\mathrm{T}_{c}$ material $\mathrm{Nd}_{2-x} \mathrm{Ce}_{x} \mathrm{CuO}_{2-y}$ (NCCO). The region of the spectrum examined by these authors $\left(50-10000 \mathrm{~cm}^{-1}\right)$ is very rich in absorption features: they observe is a "Drude-like" component at the lowest frequencies, and a set of sharp absorption peaks related to phonons and infrared active modes (IRAV, up to about $1000 \mathrm{~cm}^{-1}$ ) possibly associated to small (Holstein) polarons [32]. Three distinct absorption bands can be distinguished: the 'd-band' (around $1000 \mathrm{~cm}^{-1}$ ), the Mid-Infrared band (MIR, around $5000 \mathrm{~cm}^{-1}$ ) and the Charge-Transfer band (CT, around $10^{4} \mathrm{~cm}^{-1}$ ) [8]. Of all these features, the d-band and, at a higher temperatures, the Drude-like component have (hypothetically) been associated with large polaron optical absorption [5, [7, 14].

For the lowest levels of Ce doping, the d-band can be most clearly distinguished from the other features. The experimental optical absorption spectrum (up to $3000 \mathrm{~cm}^{-1}$ ) of $\mathrm{Nd}_{2} \mathrm{CuO}_{2-\delta}(\delta<0.004)$, obtained by Calvani and co-workers [5], is shown in Fig. 4 (shaded area) together with the theoretical curve obtained by the present method (full, bold curve) 
and, for reference, the one-polaron optical absorption result (dotted curve). At lower frequencies (600-1000 $\left.\mathrm{cm}^{-1}\right)$ a marked difference between the single polaron optical absorption and the many-polaron result is manifest. The experimental d-band can be clearly identified, rising in intensity at about $600 \mathrm{~cm}^{-1}$, peaking around $1000 \mathrm{~cm}^{-1}$, and then decreasing in intensity above that frequency. At a density of $n=1.510^{17} \mathrm{~cm}^{-3}$, we found a remarkable agreement between our theoretical predictions and the experimental curve. The experimentally determined material parameters used in the present calculation are summarized in Table I. A background contribution, taken to be constant over the frequency range of the d-band, was substracted in Figure 4. The lack of experimental data on several material constants leaves us with three adjustable parameters: the electron-phonon coupling constant $\alpha$, the band mass $m_{\mathrm{b}}$, and the density of charge carriers. These parameters were chosen as follows:

- A set of theoretical optical absorption spectra were generated for different values of the band mass and densities (for $m_{\mathrm{b}}=0.1,0.2,0.5,0.8,1.0,2.0$ and $n=$ $\left.\{0.1,0.2,0.5,1.0,1.2,1.5,2.0\} \times 10^{17} \mathrm{~cm}^{-3}\right)$.

- For each of those spectra, the coupling constant $\alpha$ was chosen so as to fit the tail region of the experimental optical absorption spectrum best, using a least squares fitting procedure (the tail region is relatively insensitive to many-polaron effects).

- The best fitting curve, using again a least squares evaluation of the goodness of fit, was selected; we found fair agreement with $m_{\mathrm{b}}=0.5 m_{\mathrm{e}}$ and $n=1.510^{17} \mathrm{~cm}^{-3}$ at $\alpha=2.1$.

This comparison with experiment could not be performed at higher doping content: the frequency region of the d-band usually contains a strong non-uniform contribution of other optical absorption features (such as the onset of the MIR, the tail of the Drude contribution, and the IRAV and phonon modes). To take these other contributions into account, additional adjustable parameters would have to be introduced making a comparison 
less convincing. Fortunately, experimental results are available in the form of the normalized first frequency moment of the optical absorption spectrum (after substraction of MIR and CT band) $\operatorname{Re}\left[\sigma_{\exp }(\omega)\right]$ :

$$
\langle\omega\rangle=\frac{\int_{0}^{\omega_{\max }} \omega \operatorname{Re}\left[\sigma_{\exp }(\omega)\right] d \omega}{\int_{0}^{\omega_{\max }} \operatorname{Re}\left[\sigma_{\exp }(\omega)\right] d \omega}
$$

where $\omega_{\max }=10000 \mathrm{~cm}^{-1}$ [5]. Calvani and co-workers [5] determined $\langle\omega\rangle$ for NCCO samples with a varying cerium doping content. Increasing the cerium doping will inject electrons in the copper-oxide planes of the material, and increase the $2 \mathrm{D}$ charge carrier density in these planes. A comparison of this experimental normalized first frequency moment to the theoretical one presents the advantage that fewer parameters need to be adapted: only the density and the electron band mass have to be taken from experiment or (if experimental values are lacking) fitted.

The carrier density can be estimated numerically from the effective carrier concentrations in the different samples [5] and from a measurement of the two-dimensional Fermi velocity performed for one of the samples [13]. As for the other cuprates, the band mass of the electrons in NCCO has not yet been determined experimentally [33, and remains as an adjustable parameter.

Fig. 5 represents the comparison between the present theory and experiment. The squares with error bars show the experimental results for differently doped samples of NCCO, reported in [5]. The dashed curve shows the normalized first frequency moment of the theoretical optical absorption spectrum, integrated over the entire frequency range $\left(\omega_{\max } \rightarrow\right.$ $\infty)$. The tail region of the many-polaron optical absorption still carries a significant weight, just as it does in the one-polaron optical absorption. It is necessary to include the cutoff frequency. The full curve represents the theoretical first frequency moment with a cutoff frequency $\omega_{\max }=10000 \mathrm{~cm}^{-1}$, which corresponds to the experimental cutoff [5].

There exists a fair agreement between the theoretical and the experimental values of the 
normalized first frequency moment for the five samples with lowest density, which have a cerium doping content of $x<0.12$. These correspond to the squares to the left of the dotted vertical line in Fig. 5. For the four remaining samples $(x>0.12)$ a discrepancy between the theoretically predicted first frequency moment for unpaired polarons and the observed

first frequency moment appears. It has been observed experimentally that the weight of the low-frequency component in these samples (with $x>0.12$ ) is significantly larger that the corresponding weight in samples with $x<0.12$ [8]. This was interpreted in [8] as a consequence of an insulator-to-metal transition taking place around the cerium-doping level of $x=0.12$. Therefore, it seems reasonable to assume that above this doping level $x$ a change in the nature of the charge carriers takes place. One could hypothesize that, as the formation of bipolarons is stabilized with increasing density of the polaron gas [34], bipolarons start playing a role in the optical absorption spectrum. In a variety of other cuprates and manganates, the presence of bipolarons has also been invoked to interpret a number of response-related properties [35].

\section{CONCLUSIONS}

Starting from the many-polaron canonical transformations and the variational manypolaron wave function (LDB) introduced in [2] we have derived a formula for the optical absorption coefficient $\operatorname{Re}[\sigma(\omega)]$ of a many-polaron gas. We find that $\operatorname{Re}[\sigma(\omega)]$ can be expressed in a closed analytical form in terms of the dynamical structure factor $S(q, \omega)$ of the electron (or hole) system, equation (24) in 2D and (23) in 3D. In the present approach, the electron-phonon coupling and the electron-electron many-body effects formally decouple in the expression for $\operatorname{Re}[\sigma(\omega)]$. Therefore, the many-body effects in the electron (hole) system can be taken into account by employing any desired approximation to the dielectric response (Hartree-Fock, RPA, etc.) of this electron (hole) system.

In the present work, the dynamical structure factor $S(q, \omega)$ of the electron (or hole) gas was considered both in the Hartree-Fock and the RPA approximation. 
The main effect of the Pauli exclusion principle on the optical absorption of the polaron gas turns out to be a shift of the oscillator strength towards higher frequencies. This effect can be understood in terms of the available initial and final states in the polaron-photon scattering process and naturally invokes the Fermi energy $E_{\mathrm{F}}$ of the electron (hole) gas.

The main effects in the case of the RPA approximation are an overall reduction of the optical absorption at frequencies $\omega>\omega_{\mathrm{LO}}$ and the introduction of a novel absorption feature which we identified as a plasmon-phonon peak. This plasmon-phonon peak shifts to higher frequencies with increasing density, such that a double peak structure can appear in the 3D many-polaron optical absorption spectrum, consistent with the observed bimodal polaronic band in cadmium oxide [36].

As a first application of the method presented here, we chose to investigate the optical absorption of the interacting polaron gas in the RPA framework. For $\mathrm{Nd}_{2} \mathrm{CuO}_{2-\delta}(\delta<0.004)$, similarities were observed (see Fig. 4) between the line shape of the experimental d-band and the many-polaron optical absorption as calculated here. To study the density dependence, measurements (performed by Calvani and co-workers [5] for a family of $\mathrm{Nd}_{2-x} \mathrm{Ce}_{x} \mathrm{CuO}_{4-y}$ materials) of the first frequency moment of the optical absorption were compared to the

results of the present theory. We find a fair agreement for the samples with the lowest densities (cerium doping $x<0.12$ ). A softening of the first frequency moment of the optical absorption for the samples with higher densities (cerium doping $x>0.12$ ) is consistent with a change in the nature of the charge carriers at a doping content $x=0.12$ inferred in ref. [8] from infrared absorption experiments.

\section{ACKNOWLEDGMENTS}

The authors like to acknowledge S. N. Klimin and V. M. Fomin for helpful discussions and intensive interactions. We are indebted to P. Calvani for fruitful discussions and for communication of experimental data. We thank F. Brosens and L. F. Lemmens for discussions. One of us, J.T., ("Postdoctoraal Onderzoeker van het Fonds voor Wetenschappelijk 
Onderzoek - Vlaanderen"), is supported financially by the Fonds voor Wetenschappelijk Onderzoek - Vlaanderen (Fund for Scientific Research - Flanders). Part of this work is performed in the framework of the "Interuniversity Poles of Attraction Program - Belgian State, Prime Minister's Office - Federal Office for Scientific, Technical and Cultural Affairs" ("Interuniversitaire Attractiepolen - Belgische Staat, Diensten van de Eerste Minister - Wetenschappelijke, Technische en Culturele Aangelegenheden"), and in the framework of the FWO projects 1.5.545.98, G.0287.95, 9.0193.97, WO.025.99N and WO.073.94N (Wetenschappelijke Onderzoeksgemeenschap, Scientific Research Community of the FWO on "Low Dimensional Systems"), and in the framework of the BOF NOI 1997 and GOA BOF UA 2000 projects of the Universiteit Antwerpen. 


\section{REFERENCES}

[1] J. T. Devreese, J. De Sitter, M. Goovaerts, Phys. Rev. B 5, 2367 (1972); see also J. T. Devreese, Polarons in Encyclopedia of Applied Physics (vol. 14, ed. G. L. Trigg, VCH, New York, 1996).

[2] L. F. Lemmens, J. T. Devreese, and F. Brosens, Phys. Stat. Sol. (b) 82, 439 (1977).

[3] G. Iadonisi, M. L. Chiofalo, V. Cataudella, and D. Ninno, Phys. Rev. B 48, 12966 (1993); V. Cataudella, G. De Filippis, and G. Iadonisi, Eur. Phys. J. B 12, 17 (1999).

[4] J. T. Devreese, S. N. Klimin, V. M. Fomin, and F. Brosens, Solid State Commun. 114, $305(2000)$.

[5] S. Lupi, P. Maselli, M. Capizzi, P. Calvani, P. Giura and P. Roy, Phys. Rev. Lett. 83, 4852 (1999).

[6] i.a. L. Genzel et al., Phys. Rev. B 40, 2170 (1989); B. Bucher et al., Phys. Rev. B 45, 3026 (1992); P. Calvani et al., Europhys. Lett. 31, 473 (1995).

[7] J. P. Falck, A. Levy, M. A. Kastner, and R. J. Birgeneau, Phys. Rev. B 48, 4043 (1993); P. Calvani et al., Solid State Commun. 91, 113 (1994).

[8] S. Lupi, P. Calvani, M. Capizzi, P. Maselli, W. Sadowski, and E. Walker, Phys. Rev. B 45, 12470 (1992).

[9] P. Calvani, M. Capizzi, S. Lupi, P. Maselli, A. Paolone, and P. Roy, Phys. Rev. B 53, 2756 (1996); S. Lupi, M. Capizzi, P. Calvani, B. Ruzicka, P. Maselli, P. Dore, and A. Paolone, Phys. Rev. B 57, 1248 (1998).

[10] M. K. Crawford, G. Burns, G. V. Chandrasekhar, F. H. Dacol, W. E. Farneth, E. M. McCarron III, and R. J. Smalley, Phys. Rev. B 41, 8933 (1990).

[11] J.-G. Zhang, X.-X. Bi, E. McRae, P. C. Ecklund, B. C. Sales, M. Mostoller, Phys. Rev. B 43, 5389 (1991). 
[12] G. A. Thomas, D. H. Rapkine, S.-W. Cheong, and L. F. Schneemeyer, Phys. Rev. B 47, 11369 (1993).

[13] C. C. Homes, B. P. Clayman, J. L. Peng, R. L. Greene, Phys. Rev. B 56, 5525 (1997).

[14] J. T. Devreese and J. Tempere, Solid State Commun. 106, 309 (1998).

[15] D. Emin, Phys. Rev. B 48, 13691 (1993).

[16] S. Tomonaga, Prog. Theoret. Phys. 2, 6 (1947); T. D. Lee, F. E. Low, and D. Pines, Phys. Rev. 90, 297 (1953).

[17] T. D. Lee, L. F. Low, D. Pines, Phys. Rev. 90, 297 (1953).

[18] See e.g. G. D. Mahan, Many-Particle Physics (Plenum Press, New York, 1993), p. 210.

[19] J. T. Devreese, in Polarons in Ionic Crystals and Semiconductors (ed. J. T. Devreese, North Holland Publ. Co., New York, 1972).

[20] See e.g. G. D. Mahan, ibid ref. [18], p. 153.

[21] J. J. Hopfield, Phys. Rev. 139, A419 (1965).

[22] A. Ron and N. Tzoar, Phys. Rev. 131, 12 (1963).

[23] V. L. Gurevich, I. G. Lang, and Yu. A. Firsov, Soviet Physics - Solid State 4, 918 (1962).

[24] G. D. Mahan, Phys. Rev. 145, 602 (1966).

[25] W. Huybrechts, J. T. Devreese, Phys. Rev. B 8, 5754 (1973).

[26] X. Wu, F. M. Peeters, and J. T. Devreese, Phys. Rev. B 31, 3420 (1985); F. M. Peeters and J. T. Devreese, Phys. Rev. B 36, 4442 (1987).

[27] X. Wu, F. M. Peeters, and J. T. Devreese, Phys. Rev. B 34, 2621 (1986); X. Wu, F. M. Peeters, and J. T. Devreese, Phys. Stat. Sol. (b) 133, 229 (1986). 
[28] M. Cardona, J. Phys. Chem. Solids 24, 1543 (1963); R. J. Collins and D. A. Kleinman, J. Phys. Chem. Solids 11, 190 (1959); W. Baer, Phys. Rev. 154, 785 (1967); see also E. Kartheuser, in Polarons in Ionic Crystals and Semiconductors (ed. J. T. Devreese, North Holland Publ. Co., New York, 1972).

[29] P. Calvani, private communication, see also Alonso, S. Tortosa, M. Garriga, and S. Piñol, Phys. Rev. B 55, 3216 (1997).

[30] For example A. Isihara, Electron Liquids (Spinger-Verlag, Berlin, 1993) or G. D. Mahan, Many-Particle Physics (Plenum Press, New York, 1993).

[31] J. T. Devreese, L. F. Lemmens, and J. Van Royen, Phys. Rev. B 15, 1212 (1977).

[32] S. Fratini, F. de Pasquale, and S. Ciuchi, e-print cond-mat/0009355.

[33] J. T. Devreese, in Models and Phenomenology for Conventional and High-Temperature Superconductivity (eds. G. Iadonisi , J.R. Schrieffer and M.L. Chiofalo, IOS Press, Amsterdam, 1999), pp. 287-304.

[34] A.A. Shanenko, M.A. Smondyrev and J.T. Devreese, Solid State Commun. 98, 1091 (1996).

[35] A. S. Alexandrov, V. V. Kabanov, and N. F. Mott, Phys. Rev. Lett. 77, 4796 (1996); A. S. Alexandrov and A. M. Bratkovsky, Phys. Rev. B 60, 6215 (1999); D. Emin, Phys. Rev. B 45, 5525 (1992); A. S. Alexandrov, J. Ranninger, Phys. Rev. B 23, 1796 (1981).

[36] H. Finkenrath, N. Uhle, and W. Waidelich, Solid State Commun. 7, 11 (1969). 


\section{TABLE}

Table I: Material parameters used in the various figures. The physical parameters for GaAs correspond to those of the GaAs-AlGaAs heterostructure [27]; the material parameters for $\mathrm{ZnO}$ are taken from [28]. The physical parameters for the neodymium-cerium cuprate are taken from [8] and [29]. "n.a." ("not applicable") means that not enough data are available to estimate this material parameter.

\begin{tabular}{lllll}
\hline \hline material parameters: & GaAs & $\mathrm{ZnO}$ & $\mathrm{Nd}_{1.85} \mathrm{Ce}_{0.15} \mathrm{CuO}_{2}$ \\
\hline phonon frequency & $\hbar \omega_{\mathrm{LO}}=36.77 \mathrm{meV}$ & $73.27 \mathrm{meV}$ & $74 \mathrm{meV}$ \\
dielectric constants & $\varepsilon_{0}=$ & 12.83 & 8.15 & n.a. \\
& $\varepsilon_{\infty}=$ & 10.9 & 4.00 & ca. 3. \\
band mass & $m_{\mathrm{b}}=$ & $0.0657 m_{\mathrm{e}}$ & $0.24 m_{\mathrm{e}}$ & n.a. \\
coupling constant & $\alpha=$ & 0.068 & 0.849 & n.a. \\
polaron length unit & $a_{\mathrm{HO}}=$ & $5.616 \mathrm{~nm}$ & $2.082 \mathrm{~nm}$ & n.a. \\
bohr radius & $a_{\mathrm{B}}=$ & $8.7797 \mathrm{~nm}$ & $0.882 \mathrm{~nm}$ & n.a. \\
\hline \hline
\end{tabular}

\section{FIGURE CAPTIONS}

Figure 1 : The real part of the optical conductivity (proportional to the optical absorption coefficient) of an interacting large-polaron gas is shown as a function of frequency, for a two dimensional gas (GaAs) from equation 24. The material parameters are given in Table I. The dashed curve represents the one-polaron result, the full curve labeled 'Hartree-Fock structure factor' shows the result using the Hartree-Fock approximation to the dynamical structure factor of the electron (hole) system, and the full curve labeled 'RPA structure factor' is the result in the Random Phase approximation. The dotted vertical line indicates the threshold frequency above which all polarons can be scattered into unoccupied final states and participate in the absorption process. The broad gray peak in the RPA curve is the plasmon-phonon contribution (see also figure 2). 
Figure 2 : The real part of the optical conductivity is shown as a function of frequency for an interacting large-polaron gas in the 2D case (left panel) and the 3D case (right panel). The material parameters used here are given in Table I. The dashed curves represent the single polaron spectra. The full curve represents the many-polaron spectrum. In this figure, the plasmon-phonon contribution to the optical many-polaron spectrum is shown as a shaded area. This contribution arises from a process where a polaron, with the absorption of a photon, emits a phonon and a plasmon. The inset shows the regions in the $q-v$ plane where the dynamical structure factor $S(q, v)$ of the electron (or hole) system used in the optical absorption formulas (23,24) differs from zero; the Landau damping region and the undamped plasmon branch can be distinguished.

Figure 3: The real part of the optical conductivity is shown as a function of frequency for different densities of an interacting large-polaron gas, in the 2D case (left panel) and the $3 \mathrm{D}$ case (right panel). The material parameters used for this figure are given in Table I. For increasing density, the optical conductivity is reduced. Another effect in the RPA approximation is the presence of a peak related to the undamped plasmon branch (see figure 2) which shifts according to the plasma frequency.

Figure 4 : The infrared absorption of $\mathrm{Nd}_{2} \mathrm{CuO}_{2-\delta}(\delta<0.004)$ is shown as a function of frequency, up to $3000 \mathrm{~cm}^{-1}$. The experimental results of Calvani and co-workers [5] is represented by the thin black curve and by the shaded area. The so-called 'd-band' rises in intensity around $600 \mathrm{~cm}^{-1}$ and increases in intensity up to a maximum around 1000 $\mathrm{cm}^{-1}$. The dotted curve shows the single polaron result. The full black curve represents the theoretical results obtained in the present work for the interacting many-polaron gas with $n=1.510^{17} \mathrm{~cm}^{-3}, \alpha=2.1$ and $m_{\mathrm{b}}=0.5 m_{\mathrm{e}}$.

Figure 5 : The normalized first frequency moment of the optical absorption spectra is shown as a function of the density (expressed through the Fermi wave vector). The squares represent the experimental results of Calvani and co-workers [5] in a family of $\mathrm{Nd}_{2-x} \mathrm{Ce}_{x} \mathrm{CuO}_{4}$ materials. The dashed curve shows the results from the theoretical two-dimensional manypolaron optical absorption, obtained by integrating all frequencies in the calculation of the 
first frequency moment. The full curve shows the theoretical results obtained by integrating up to a cut-off frequency, which is chosen at $10000 \mathrm{~cm}^{-1}$ and which corresponds to the maximum frequency in the experiment [5]. The material parameters are listed in the figure, and the effect of choosing a different electron band mass is illustrated in the inset. The points with $x<0.12$, to the left of the vertical dotted line, show agreement with the theoretical result from the many-polaron theoretical optical absorption, but it is clear that the experimental cutoff frequency has to be taken into account. For the samples with $x>0.12$, a discrepancy between the theoretically predicted first frequency moment and the observed first frequency moment is consistent with a possible insulator-to-metal transition at $x=0.12$ [8]. 


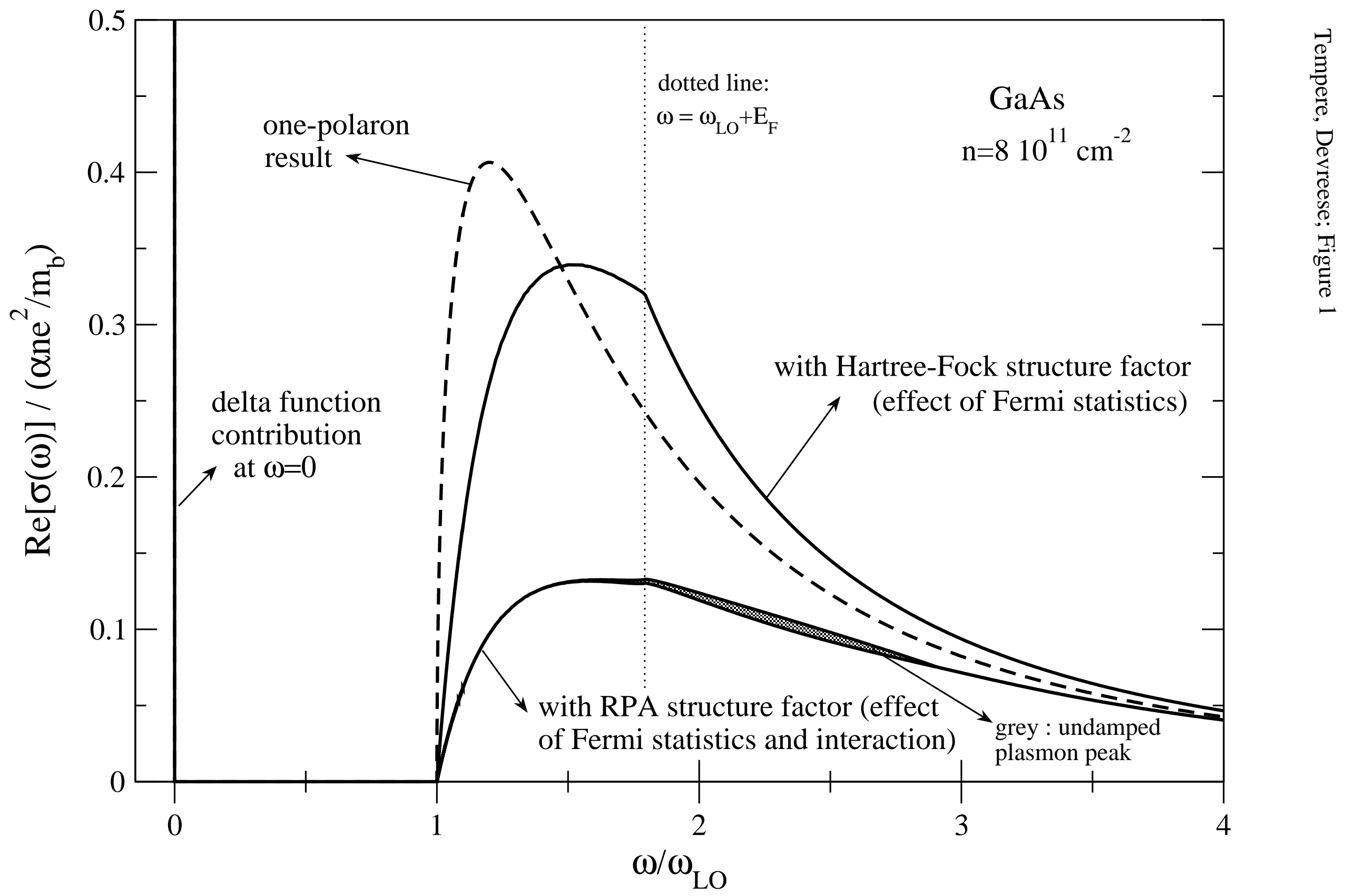


2D optical absorption for GaAs

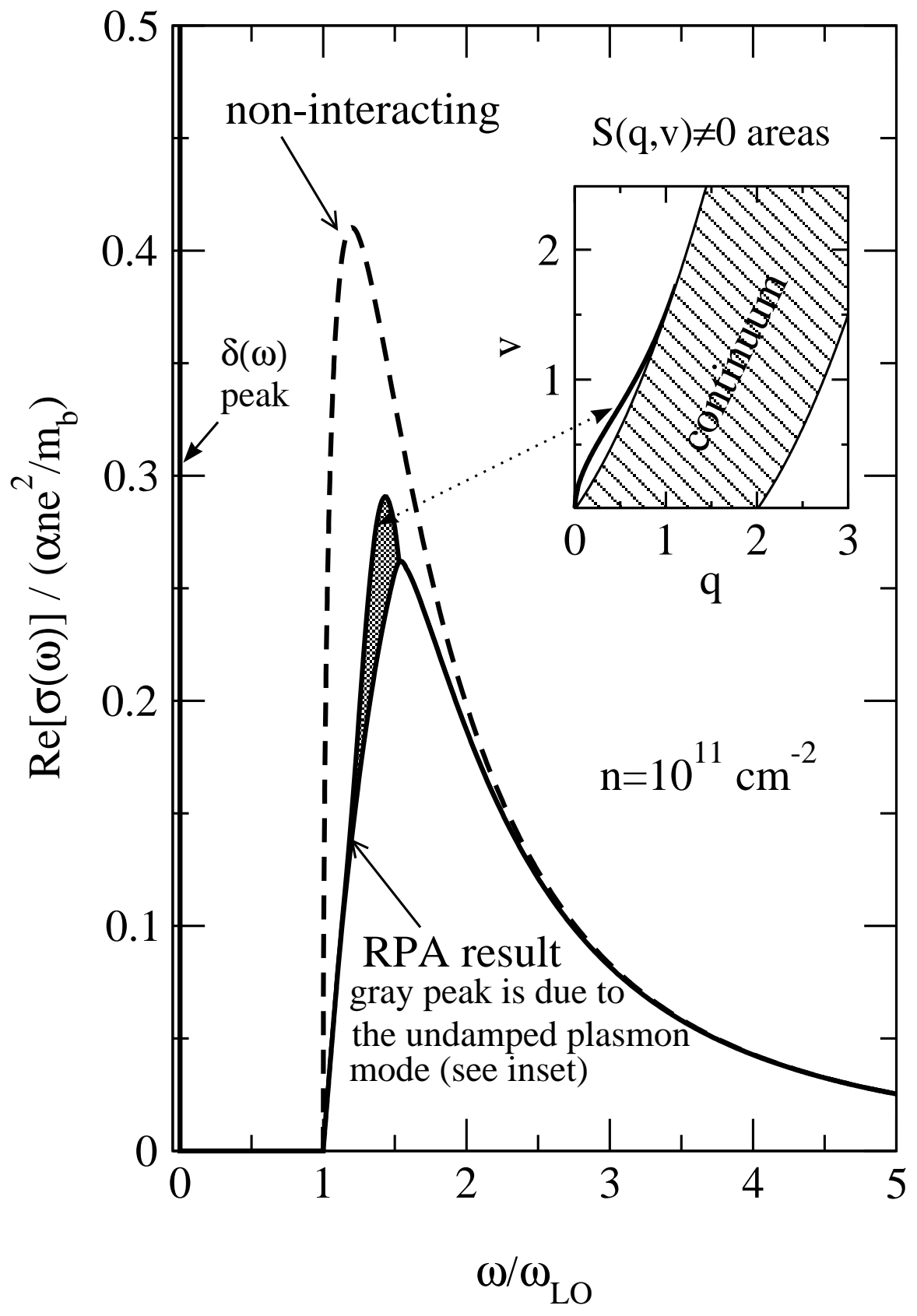

3D optical absorption for $\mathrm{ZnO}$

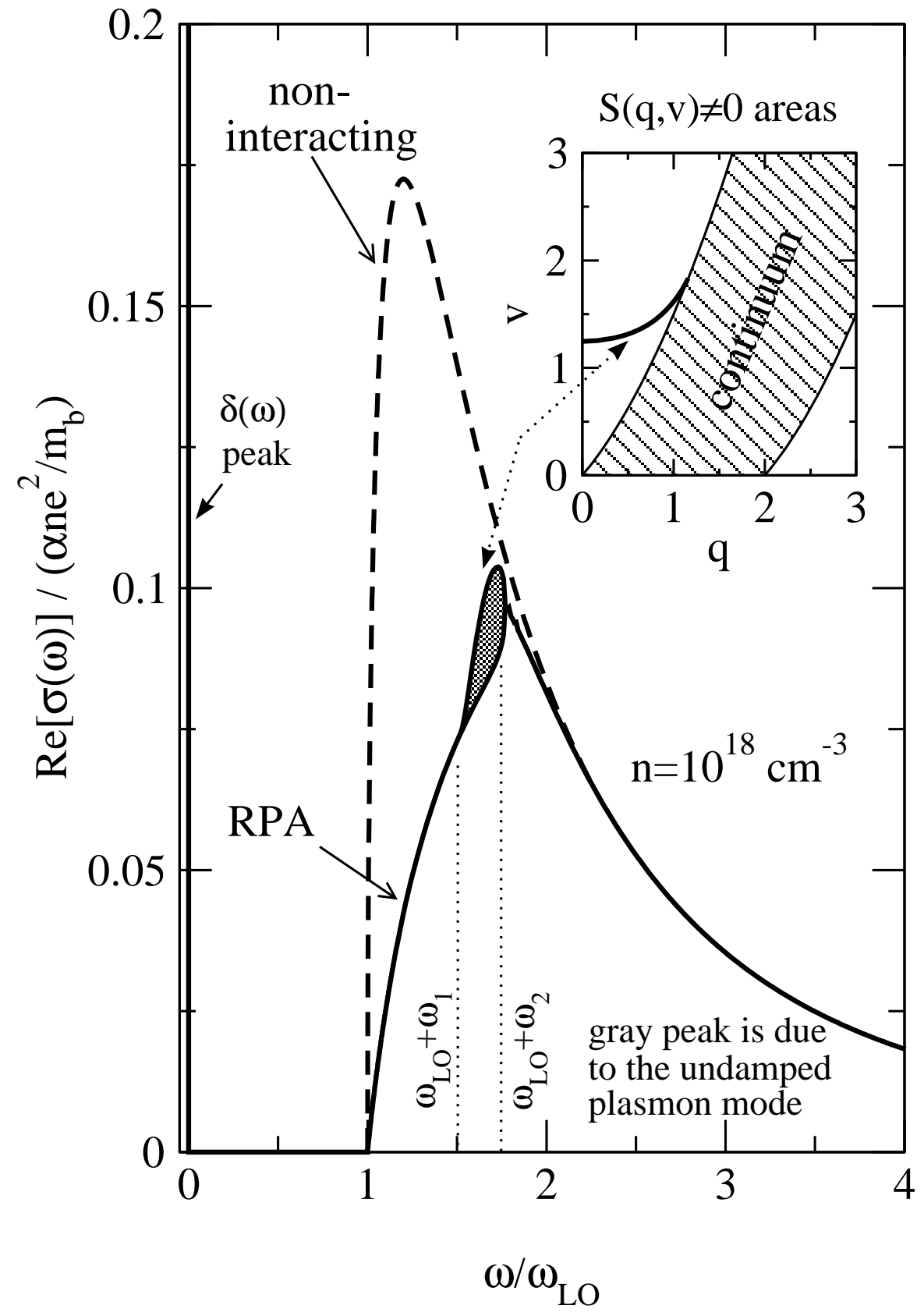


Tempere, Devreese; Figure 3
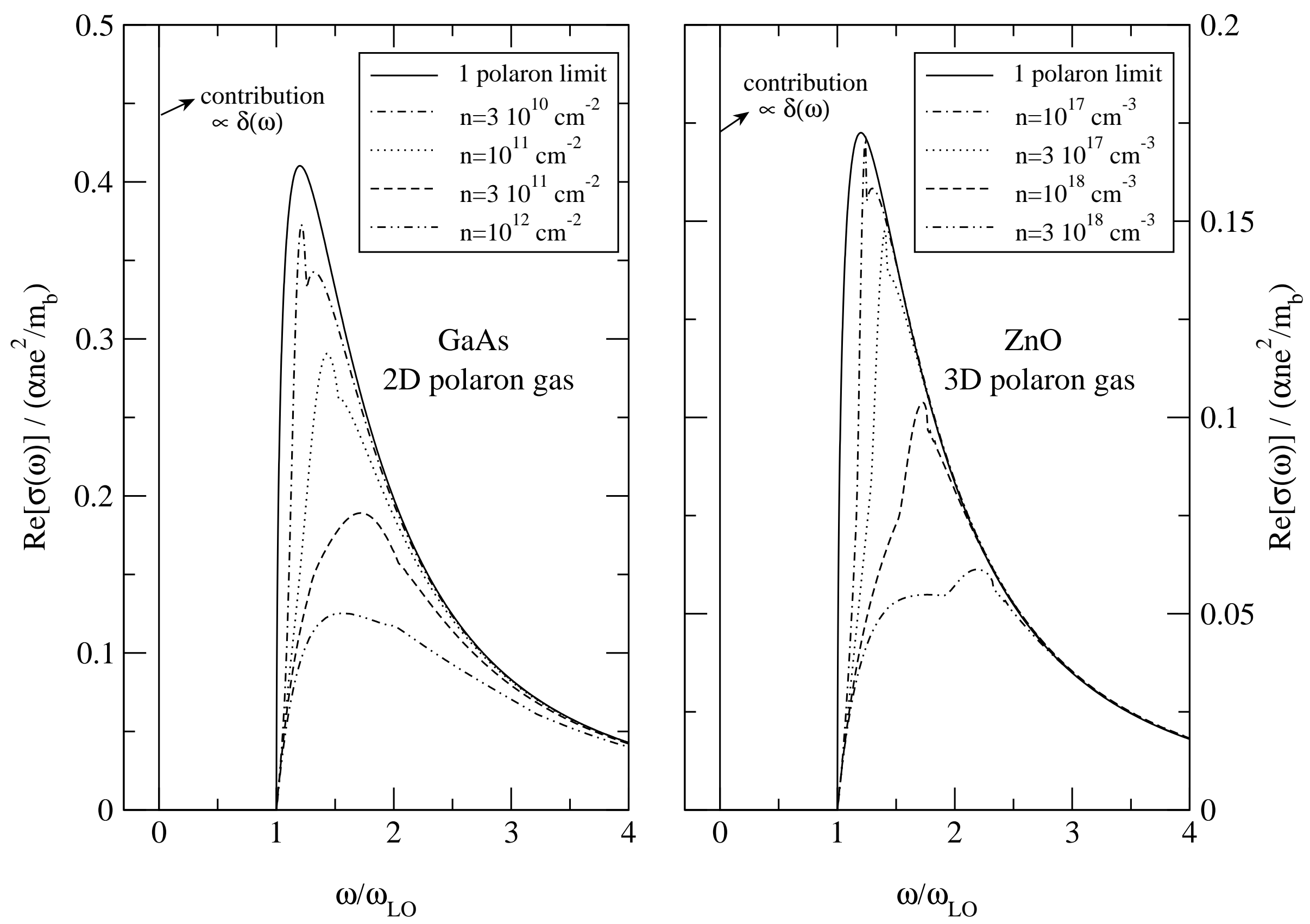
Tempere, Devreese; Figure 4

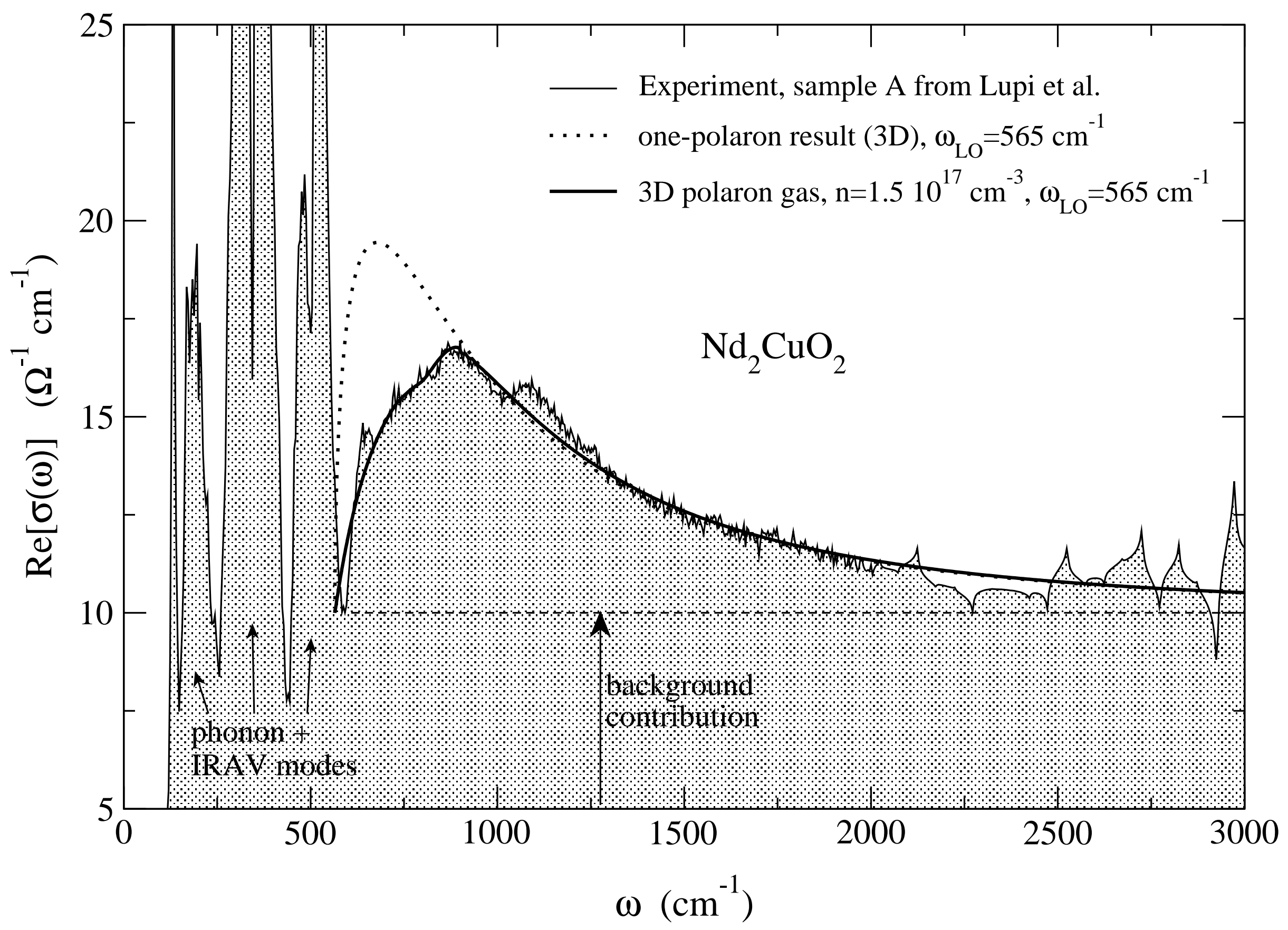


Tempere, Devreese; Figure 5

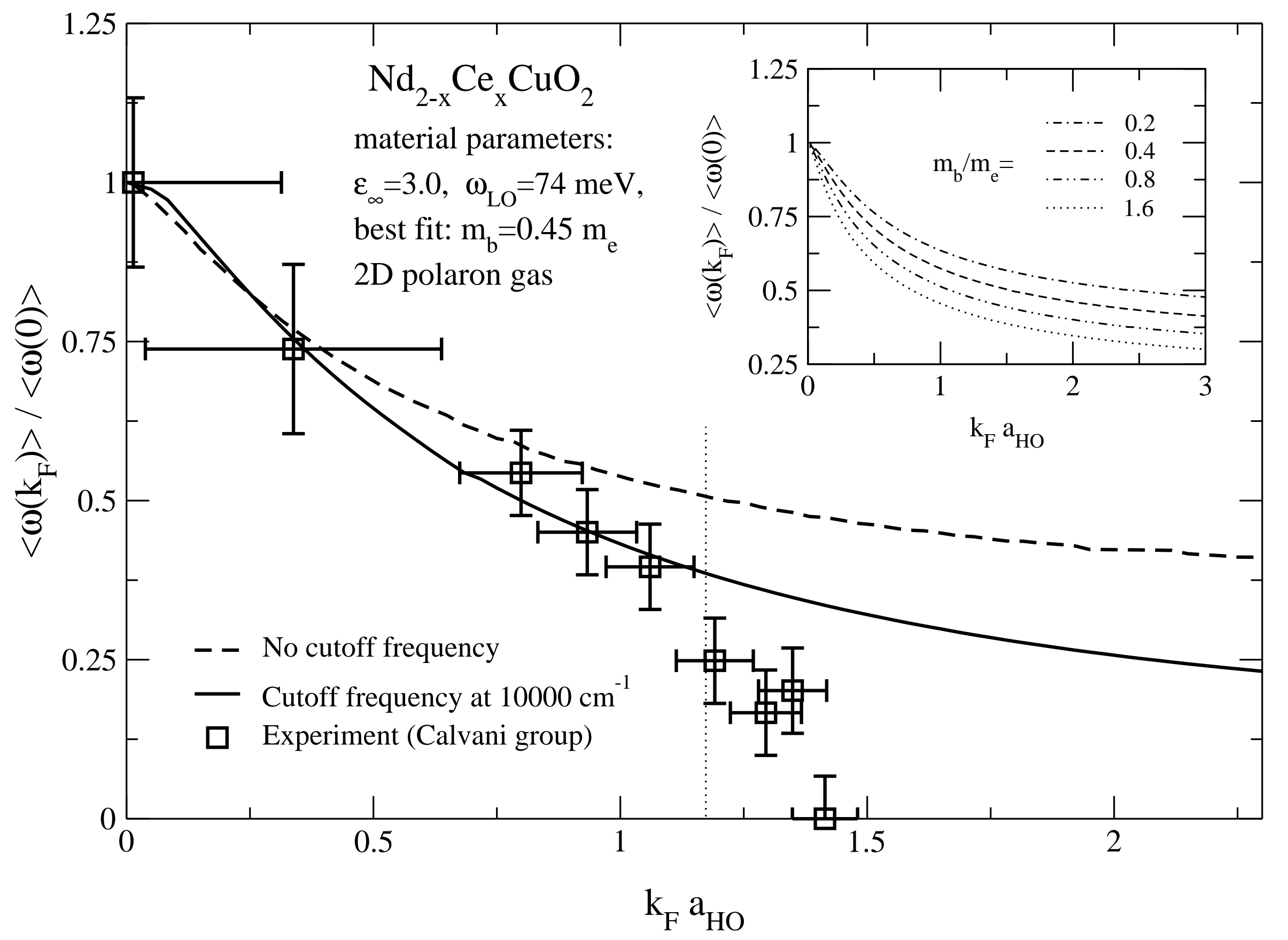

